\title{
Component duration effects in multiple schedules
}

\author{
BEN A. WILLIAMS \\ University of California, San Diego, La Jolla, California
}

\begin{abstract}
Previous research has produced conflicting results regarding the effects of component duration on interactions in multiple schedules. In Experiment 1, potential sources of this conflict were evaluated. Both the effects of absolute reinforcement rate and carry-over effects (hysteresis) from a preceding condition were isolated. When 10 -sec components were used, the sensitivity of relative response rate to relative reinforcement rate was affected very little by hysteresis effects and absolute reinforcement rate, but it was systematically reduced as a function of the number of prior conditions. Sensitivity to relative reinforcement rate was also substantially higher with the 10-sec components than with 2-min components. In Experiment 2, this effect of component duration was decomposed into two separate effects. Contrast effects during presentation of a target component with a constant reinforcement rate were greater the shorter the target component was itself; but they were smaller the shorter the alternative component in which reinforcement rate was varied. The latter effect was smaller and more unreliable across subjects. The existence of these two separate effects demonstrates that the usual method of studying component duration-that is, holding all components equal in duration-systematically causes underestimation of the effects of the component duration, and obscures the different processes controlling the two effects.
\end{abstract}

A major emphasis in free-operant research has been the development of quantitative descriptions of behavior. One of the most influential of such descriptions is the generalized matching law, given by Equation 1, which has been applied to schedules in which two components are available, either simultaneously or successively $(B$ refers to the behavior in a component; $R$ refers to the corresponding reinforcement rate; $b$ refers to a bias independent of the reinforcement rates, and $a$ to the sensitivity to relative reinforcement rate). The assumption underlying this application is that variation in the interactions between the components of the schedule can be captured adequately by the two parameters of the expression. For this assumption to be sustained, parameter variations that should have important effects on behavior should yield corresponding changes in either bias or sensitivity.

$$
B_{1} / B_{2}=b\left(R_{1} / R_{2}\right)^{a}
$$

An important schedule parameter that seems to challenge the use of Equation 1 as a description of multiple schedule behavior is the duration of the schedule components. Given that contrast effects in one component of the schedule are an inverse function of the reinforcement rate during the alternative component (see Williams, 1983a, for a review), it seems intuitively plausible that the duration of the components should be an important determinant of the size of the contrast effects. When each

This research was supported by NSF Grant BNS84-08878 and NIMH Grant I RO1 MH42797. Requests for reprints should be addressed to Ben A. Williams, Department of Psychology, C-009, University of California, San Diego, La Jolla, CA 92093 . Data for individual subjects from individual conditions are also available from the author upon request. component is long, responding at most points in the component is distant from the alternative reinforcement schedule; when each component is short, responding at any point is close to the alternative schedule. It seems plausible, therefore, that the size of contrast effects should be greater with shorter components. Consequently, the exponent value of Equation 1 should be larger as well.

Despite this intuition, the available evidence regarding the effect of component duration on multiple schedule interactions, as assessed by Equation 1, yields a confused picture. Early evidence (Shimp \& Wheatley, 1971; Todorov, 1972) showed larger interactions with shorter components. Fits of Equation 1 by McSweeney, Farmer, Dougan, and Whipple (1986) to the subjects of Shimp and Wheatley (1971) yielded median values of $a$ that ranged from .86 to .52 as component duration varied from 2 to $180 \mathrm{sec}$. Thus, a strong effect of component duration occurred, and values of $a$ with the shortest components were comparable to those typically obtained with concurrent schedules.

Later studies have challenged the generality of these findings. Charman and Davison (1982) failed to show any effect of component duration over the range of $5 \mathrm{sec}$ to 6 min when several different reinforcement schedules were presented, and, when using short component durations comparable to those in the earlier studies, they found exponent values much smaller. For example, when using 5-sec components, Charman and Davison (Experiment 2), reported values of the exponent in the range of .27 to .90 (median of .46), which, as noted above, were not significantly different from those found with a collection of longer component durations. Similarly, using 15-sec components, White et al. (1985) reported exponent values of .30 to .43 (median of .32 ), which was not 
different from their values obtained with 60 -sec components.

The failure to find any effect of component duration in the later studies is surprising, given that several studies have shown greater contrast effects with shorter components when the measure was the change in absolute response rate rather than the exponent of Equation 1 (Hinson, Malone, McNally, \& Rowe, 1978; McSweeney, 1982; Schwartz, 1978; Spealman, 1976; Williams, 1980). The issue raised is whether this difference says something important about the limitations of Equation 1 as a description of behavior (but see White, Pipe, McLean, \& Redman, 1985 , for a hypothesis for why the different measures should yield different outcomes).

Clarification of the status of component-duration effects is important for reasons other than to ascertain the adequacy of the generalized matching law as a description of multiple-schedule behavior. Such effects are also important because they impinge strongly on the issue of how performance on different types of schedules should be conceptualized. As has been noted by McSweeney et al. (1986), a concurrent schedule might be regarded as a multiple schedule with very short components. Accordingly, the fact that concurrent schedules typically produce larger interactions than multiple schedules do would be ascribed to the effects of component duration, with the implication that robust effects of component duration should be obtained when they are studied in multiple schedules directly. Alternatively, if there is no consistent effect of component duration, there should be little basis for believing that the two schedule situations share the same determining processes.

A possible reason for the conflict in previous results was discussed by Charman and Davison (1982). They noted that their procedure differed from that of Shimp and Wheatley (1971) in terms of the number of successive conditions in which the reinforcement schedules in the two components were reversed: a large proportion of their experimental conditions involved reinforcement-rate reversals, whereas such reversals occurred infrequently for Shimp and Wheatley. Charman and Davison provided further support for the role of such reversals. They demonstrated a small effect of component duration by varying it while holding constant a single pair of schedules, in contrast to no effect when the reinforcement schedules and component duration were varied together. They further noted a lower sensitivity to relative reinforcement rate during the first condition after the schedule values were reversed for the two components of the schedule. Their results thus suggest that "hysteresis" effects may play a significant role in the attenuation of schedule interactions, and that these effects may obscure componentduration effects that would otherwise be found to occur.

\section{EXPERIMENT 1}

Hysteresis effects are a well-known feature of many psychological procedures. The standard method for as- sessing the role of these effects is to use ascending and descending series (for examples of hysteresis effects in operant research, see Baum, 1974, and Williams \& Fantino, 1978; a general discussion of the problem is provided by Stevens, 1957). That is, the comparison of the outcome of a condition as a function of whether it is part of an ascending or a descending series provides an index of the effect of preceding conditions, which can then be factored out. Experiment 1 included this procedural feature in the attempt to resolve the disparity between previous results.

A second variable studied in Experiment 1 was the absolute rate of reinforcement, summed over the two schedules. Previous studies have typically varied both the relative and the absolute reinforcement rates simultaneously, so that it has been unclear whether changes in absolute reinforcement rate may play a significant role on its own, as is suggested by some theoretical models (McLean \& White, 1983). In the present study, the total reinforcement rate summed over both components, maintained at either a high or low level, was held constant for a given series of conditions. Then the same series of conditions was repeated for the alternative total level of reinforcement.

Experiment 1 was designed first to establish the sensitivity of relative response rate to relative reinforcement rate in short-component $(10-\mathrm{sec})$ multiple schedules, in which the effects of hysteresis and absolute response rate were isolated. Then it was determined whether or not this sensitivity changed as a function of changing the duration of the schedule components.

\section{Method}

Subjects. Four White Carneaux pigeons were maintained at $80 \%$ of their free-feeding weights by means of additional feeding when necessary after the end of the experimental sessions. All had previously served as subjects in an undergraduate laboratory, where they had been trained to peck a white keylight under various simple reinforcement schedules.

Apparatus. The experimental chamber was constructed from a plastic picnic chest with the metal intelligence panel inserted midway through its interior. The bird's chamber was approximately $30.5 \mathrm{~cm}$ in all dimensions. Two pigeon keys were mounted on the intelligence panel, each $1.7 \mathrm{~cm}$ in diameter and requiring a minimum force of $0.10 \mathrm{~N}$ for operation. The keys were positioned $10 \mathrm{~cm}$ apart, with the midpoint of their separation located at the center of the intelligence point, which was $10 \mathrm{~cm}$ above a $5 \times 5 \mathrm{~cm}$ aperture through which the birds could eat when the food hopper was activated and illuminated with white light. Pecks on the keys provided no feedback except for the keys' discursion (a maximum of $0.5 \mathrm{~cm}$ ) and the operation of the microswitch behind the keys. The stimuli were projected onto the rear of the keys by standard 28-V 12-stimulus IEE in-line projectors. The chamber was illuminated by a $28-\mathrm{V}$ shielded white houselight, illuminated at all times during an experimental session, mounted on the rear wall of the chamber opposite the intelligence panel at the same height as the keylights.

Procedure. Because all subjects previously had learned to keypeck, they were begun immediately on the two-component multiple schedule with alternating 10-sec components. The different components were correlated with different response keys. Component 1 was cued by a yellow keylight projected onto the right key, and Component 2 was cued by a green keylight projected onto the left 
key. During the first three sessions the schedules were VI (variable interval) $15 \mathrm{sec}$ in each component. Thereafter the subjects were trained on the order of conditions shown in Table 1 . Subjects 81 and 83 were begun on the high reinforcement-rate series; the sum of their two schedules totaled 120 reinforcers $/ h$. Subjects 84 and 85 were begun on the low reinforcement-rate series; their sum totaled 30 reinforcers $/ h$. Because of an error, the initial condition for the latter pair of subjects was an odd pair of schedules, so that the ascending and descending series for those subjects did not begin until the second condition. Consequently, their final two conditions of the first series ( 9 and 10 ) were those that otherwise would have occurred as Conditions 1 and 2.

After the ascending and descending series were completed with the original assignment of subjects to the two different values of overall reinforcement rate, the assignments were reversed, and ascending and descending series were repeated. Because of time constraints, the ascending series for Subjects 84 and 85 included only the middle and two extreme points of the series, rather than all five points.

In the final set of conditions (19-24 for Subjects 81 and 83; 18-23 for Subjects 84 and 85 ), the effects of component duration were investigated. Whereas all previous training had used 10 -sec components, this was now changed to $120 \mathrm{sec}$ for a descending series of three conditions, and was then returned to $10 \mathrm{sec}$ for an ascending series through the same set of conditions.

Experimental sessions continued for 50-55 min for all conditions. The VI schedules, which were constructed from the exponential distribution of Fleshler and Hoffman (1962), involved 18 separate reinforcement intervals. Training within a condition continued for a minimum of 15 sessions and until a stability criterion was reached: sessions were grouped in running blocks of three, and a relative response rate was calculated for each block. When these values differed by no more than .05 , and when there were no ascending or descending trends for three such blocks (the last nine sessions),

\section{Table 1}

Values of the VI Schedules Presented in the Two Components of Experiment 1

\begin{tabular}{|c|c|c|c|c|c|c|}
\hline \multirow[b]{2}{*}{ Order } & \multicolumn{3}{|c|}{ Subjects 81 and 83} & \multicolumn{3}{|c|}{ Subjects 84 and 85} \\
\hline & Comp. 1 & Comp. 2 & Sessions & Comp. 1 & Comp. 2 & Sessions \\
\hline 1 & $16 \mathrm{sec}$ & $4 \mathrm{~min}$ & 19 & $64 \mathrm{sec}$ & $16 \mathrm{sec}$ & 20 \\
\hline 2 & $20 \mathrm{sec}$ & $60 \mathrm{sec}$ & 24 & $80 \mathrm{sec}$ & $4 \mathrm{~min}$ & 36 \\
\hline 3 & $30 \mathrm{sec}$ & $30 \mathrm{sec}$ & 19 & $2 \min$ & $2 \mathrm{~min}$ & 28 \\
\hline 4 & $60 \mathrm{sec}$ & $20 \mathrm{sec}$ & 24 & $4 \mathrm{~min}$ & $80 \mathrm{sec}$ & 26 \\
\hline 5 & $4 \mathrm{~min}$ & $16 \mathrm{sec}$ & 15 & $16 \mathrm{~min}$ & $64 \mathrm{sec}$ & 19 \\
\hline 6 & $60 \mathrm{sec}$ & $20 \mathrm{sec}$ & 16 & $4 \mathrm{~min}$ & $80 \mathrm{sec}$ & 19 \\
\hline 7 & $30 \mathrm{sec}$ & $30 \mathrm{sec}$ & 19 & $2 \mathrm{~min}$ & $2 \mathrm{~min}$ & 31 \\
\hline 8 & $20 \mathrm{sec}$ & $60 \mathrm{sec}$ & 22 & $80 \mathrm{sec}$ & $4 \mathrm{~min}$ & 28 \\
\hline 9 & $16 \mathrm{sec}$ & $4 \mathrm{~min}$ & 19 & $64 \mathrm{sec}$ & $16 \mathrm{~min}$ & 21 \\
\hline 10 & $64 \mathrm{sec}$ & $16 \mathrm{~min}$ & 24 & $80 \mathrm{sec}$ & $4 \mathrm{~min}$ & 30 \\
\hline 11 & $80 \mathrm{sec}$ & $4 \mathrm{~min}$ & 33 & $16 \mathrm{sec}$ & $4 \mathrm{~min}$ & 23 \\
\hline 12 & $2 \min$ & $2 \min$ & 26 & $20 \mathrm{sec}$ & $60 \mathrm{sec}$ & 22 \\
\hline 13 & $4 \mathrm{~min}$ & $80 \mathrm{sec}$ & 36 & $30 \mathrm{sec}$ & $30 \mathrm{sec}$ & 18 \\
\hline 14 & $16 \mathrm{~min}$ & $64 \mathrm{sec}$ & 24 & $60 \mathrm{sec}$ & $20 \mathrm{sec}$ & 23 \\
\hline 15 & $4 \min$ & $80 \mathrm{sec}$ & 21 & $4 \mathrm{~min}$ & $16 \mathrm{sec}$ & 16 \\
\hline 16 & $2 \mathrm{~min}$ & $2 \mathrm{~min}$ & 27 & $30 \mathrm{sec}$ & $30 \mathrm{sec}$ & 15 \\
\hline 17 & $80 \mathrm{sec}$ & $4 \mathrm{~min}$ & 27 & $16 \mathrm{sec}$ & $4 \mathrm{~min}$ & 37 \\
\hline 18 & $64 \mathrm{sec}$ & $16 \mathrm{~min}$ & 23 & $16 \mathrm{sec}$ & $4 \mathrm{~min}$ & 17 \\
\hline 19 & $64 \mathrm{sec}$ & $16 \mathrm{~min}$ & 36 & $30 \mathrm{sec}$ & $30 \mathrm{sec}$ & 20 \\
\hline 20 & $2 \min$ & $2 \mathrm{~min}$ & 15 & $4 \mathrm{~min}$ & $16 \mathrm{sec}$ & 34 \\
\hline 21 & $16 \mathrm{~min}$ & $64 \mathrm{sec}$ & 21 & $4 \mathrm{~min}$ & $16 \mathrm{sec}$ & 17 \\
\hline 22 & $16 \mathrm{~min}$ & $64 \mathrm{sec}$ & 17 & $30 \mathrm{sec}$ & $30 \mathrm{sec}$ & 23 \\
\hline 23 & $2 \mathrm{~min}$ & $2 \mathrm{~min}$ & 19 & $16 \mathrm{sec}$ & $4 \mathrm{~min}$ & 32 \\
\hline 24 & $64 \mathrm{sec}$ & $16 \min$ & 35 & & & \\
\hline
\end{tabular}

Note-Component duration was always 10 sec except in Conditions 19-21 for Subjects 81 and 83, and Conditions $18-20$ for Subjects 84 and 85, when it was $2 \mathrm{~min}$.
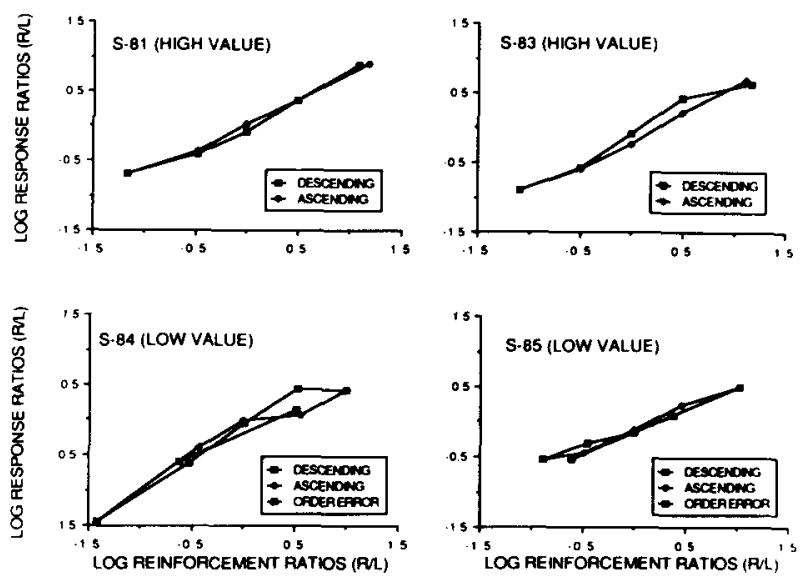

Figure 1. Data from the first series of conditions for individual subjects in Experiment 1. Separate functions are for the conditions in ascending versus descending series. The third function for Subjects 84 and 85 (S-84 and S-85) correspond to the first two conditions presented (see text for details).

the relative response rates were considered stable. In addition, the subjects were run in pairs so that both subjects in a pair had to satisfy the stability criterion before they were moved together to the next condition.

\section{Results}

Because of the large number of conditions, the absolute response rates and obtained reinforcement frequencies will not be shown (these may be obtained from the author upon request). Figure 1 shows the ratio of response rates in the two components as a function of the ratio of their reinforcement rates for the first assignment of subjects to the different levels of absolute reinforcement rate. Log values are used in order to facilitate the analysis in terms of the generalized matching law (Equation 1); the slope of the lines corresponds to the sensitivity parameter, $a$, and the $y$-intercept corresponds to the bias term, $b$, when $\log$ - $\log$ plots are used. Note that for the conditions shown in Figure 1, Subjects 81 and 83 were trained with the high reinforcement rates and Subjects 84 and 85 were trained with the low reinforcement rates. Shown separately are the ascending and descending series. Note that Subjects 84 and 85 have a third function connecting only two points, which were the first two conditions shown in Table 1. These are presented separately, because the first condition involved an error in the schedule assignments and thus was not part of the descending order. It is apparent that this function falls in approximately the same location as the other functions, so the error in order of presentation made little difference.

Hysteresis effects are indicated by the difference between the ascending and descending series; carryover from the preceding condition should cause the function for the descending series to be higher on the graph. For 3 of the 4 subjects, there is no meaningful difference between the two functions, but for the remaining subject 

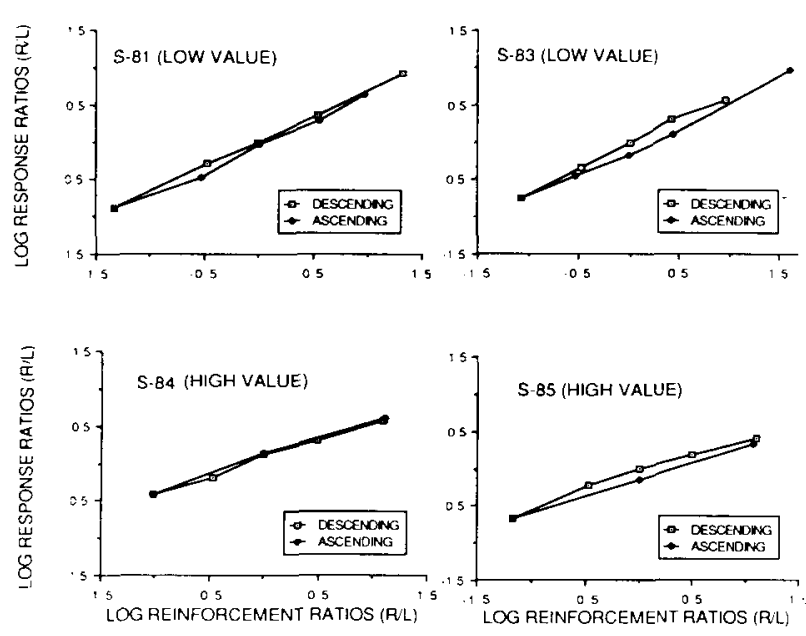

Figure 2. Data from the second series of conditions presented in Experiment 1. Separate functions are from conditions in ascending versus descending series.

(Subject 83), the descending function is slightly higher, indicating a modest hysteresis effect.

Figure 2 shows the functions for the ascending and descending series after the assignment of subjects to the different absolute reinforcement rates was reversed. Note that Subjects 84 and 85 have only three points for the ascending series, because it was abbreviated due to time constraints. As in Figure 1, there is little evidence for any hysteresis; Subject 83 again showed a modest effect, and Subject $\mathbf{8 5}$ showed a similar small effect for the one condition shared by the two series.

The data points of each of the individual functions shown in Figures 1 and 2 were fitted separately by Equation 1 , with the resulting parameter values and variance accounted for as shown in Table 2. For neither set of conditions were there any consistent changes in the best-fitting equations as a function of the ascending and descending

Table 2

Values of the Exponents for the Best Fits of Equation 1 to the Results of Experiment 1

\begin{tabular}{clccc}
\hline Subject & & High Value & Low Value & All \\
\hline 81 & $a$ & .73 & .68 & .70 \\
& $b$ & 1.05 & .96 & 1.00 \\
& VAC & 98.5 & 99.2 & 98.6 \\
83 & $a$ & .74 & .65 & .69 \\
& $b$ & .72 & .94 & .78 \\
& VAC & 97.0 & 98.2 & 97.0 \\
84 & $a$ & .48 & .78 & .66 \\
& $b$ & 1.21 & .68 & .83 \\
& VAC & 99.0 & 92.7 & 86.3 \\
& $a$ & .45 & .56 & .51 \\
& $b$ & .81 & .80 & .79 \\
& VAC & 97.2 & 97.5 & 95.8 \\
\hline
\end{tabular}

Note-Separate fits were made for each level of absolute reinforcement frequency, and then all data combined. Subjects 81 and 83 first received the high-value schedules, whereas Subjects 84 and 85 first received the low-value schedules. series, so this distinction will be ignored. For the first set of conditions (high value for Subjects 81 and 83, low value for Subjects 84 and 85 ), fits to the aggregate of both series yielded values of $a$ that ranged between .56 and .78 , with a median of .74 . The fits provided by Equation 1 were also quite good: more than $90 \%$ of the variance was accounted for (VAC) in every case, with a median of $97 \%$.

Results of the fits to the second series of conditions (low value for Subjects 81 and 83, high value for Subjects 84 and 85 ) reveal that the accuracy of the fits continued to be quite high, since a median of $98 \%$ of the variance was accounted for. Now, however, the sensitivity exponent was somewhat reduced for all the subjects, with the result that the $a$ values now ranged from .45 to .68 , with a median of .56 .

The final column of Table 2 shows the results of the fit of Equation 1 to all conditions combined. In general, the best-fitting parameter values were intermediate between those obtained for the separate fits to the high- and low-value reinforcement schedules, indicating that the mixture of different absolute rates of reinforcement was not an important variable in determining the sensitivity of relative response rate to relative reinforcement rate. The accuracy of the fits also continued to be quite high, except in the case of Subject 84. This was due to its bias term's shifting in the opposite direction between conditions with the high and low reinforcement rates.

The last observation from Table 2 concerns whether there was any difference between the parameter values as a function of the absolute frequency of reinforcement. The median value of $a$ from the fits of the high-value conditions was .61, while that from the fits to the low-value conditions was .67 . Whether this represents a meaningful difference is uncertain, given the small number of subjects and the confounding effects of order of presentation. In order to provide a more powerful test of the effects of absolute frequency of reinforcement, the data from the individual conditions for each reinforcement frequency were pooled across subjects. Thus, the experimental conditions included in each pool were counterbalanced with respect to the order of presentation of the two different reinforcement frequencies. The results of the analysis are shown in Table 3. Despite the fact that the pooling of subjects causes the different biases for individual subjects to become a source of error variance, Equation 1 continued to provide a good description of the relative response rates, for over $90 \%$ of the variance was accounted for in both cases. The estimates of $a$ for the pooled data were similar to the median values for individual subjects noted above; $a=.612$ for the high reinforcement frequencies and .685 for the low reinforcement frequency. The standard errors for the separate estimates were quite small; the .05 confidence interval for either estimate did not overlap the alternative estimate. But a more stringent test is provided by a $t$ test of the difference between the two estimates. The result was that $t(68)=1.61$, which was not significant at the .05 level. 
Table 3

Fits of Equation 1 to the Pooled Data from Individual Subjects

\begin{tabular}{lcccc}
\hline & $b$ & $a$ & $S E$ for $a$ & VAC \\
\hline High frequency & .92 & .612 & .036 & 90.4 \\
Low frequency & .80 & .685 & .028 & 94.8 \\
\hline
\end{tabular}

Note-Data are divided according to the absolute frequency of reinforcement, and are from all conditions prior to exposure to the change in component duration.

Table 4

Fits of Equation 1 to Pooled Data from All Subjects

\begin{tabular}{lllcc}
\hline Conditions & $b$ & $a$ & $S E$ for $a$ & VAC \\
\hline $1-5$ & .82 & .74 & .046 & 93.7 \\
$6-10$ & .79 & .71 & .046 & 92.9 \\
$11-15$ & .93 & .57 & .034 & 93.8 \\
$16-20(22)$ & .88 & .56 & .039 & 91.3 \\
\hline
\end{tabular}

Note-Data are subdivided into blocks of successive conditions, including all conditions with 10-sec component durations. The last block contained seven rather than five experimental conditions for Subjects 81 and 83.

The comparison of the fits to the first and second series of conditions suggests that sensitivity was reduced as a function of an increasing number of experimental conditions. Because this effect was obscured by the effects of overall reinforcement rate, some method of assessing the change independently of the changes in reinforcement rate was desirable. To accomplish this, the data from all 4 subjects were again pooled together and partitioned into blocks of five conditions. Since over 20 experimental conditions (excluding those with the 2-min components) were presented to each subject, this provided a series of four blocks over which any reduction in sensitivity could better be detected. Note that the effect of the pooling of subjects is to have each level of reinforcement rate represented approximately equally in each segment so that any effects of that variable will be canceled. The fits of Equation 1 to each block of five conditions are shown in Table 4. No consistent change in the bias term is evident, and the percentage of variance accounted for remained high throughout training. Table 4 does show, however, a monotonic drop across training in the exponent $a$ from .74 for the first block to .56 for the final block. This reduction in the value of $a$ is substantially greater than the standard errors of the estimates of $a$, also shown in Table 6 . A $t$ test conducted on the difference between the two extreme values showed it to be statistically reliable $[t(68)$ $=2.66, p<.05]$.

All of the results already considered came from conditions in which 10-sec component durations were used. In order to substantiate that sensitivity to relative reinforcement rate was a function of component duration, component duration was changed to $2 \mathrm{~min}$ for three conditions. This provided an estimate of the sensitivity with longer components. Because of the effects of prior training noted above, it was then necessary to return to the 10 -sec component durations for a final series of three conditions, to ensure that any reduction in the exponent value was due to the component duration, as opposed to the order of training. Table 5 provides the parameter values of the best fits of Equation 1 to the series with 10-sec components prior to the change to $2 \mathrm{~min}$, to the series with the 2-min components, and to the series after the return to the 10sec components. Note that these involve only the conditions corresponding to those after the change in component duration-that is, to only three points on the series rather than all five.

No consistent changes in the bias terms occurred as a function of changing the component duration. Of primary interest are the values of the exponent $a$. For all subjects, the values with the 2-min components are lower than those from both presentations of the 10-sec components; the mean value of the sensitivity exponent was .56 for the 10 -sec series prior to the 2 -min series, .53 for the 10 -sec series after the 2 -min series, and .35 for the 2 -min series itself.

\section{Discussion}

The present results show that the determinants of schedule interactions in multiple schedules are complex and not easy to understand. On the basis of the results of Charman and Davison (1982), the expectation was that hysteresis effects were a major cause of decreased sensitivity to relative reinforcement rate in multiple schedules, and that the use of ascending and descending series would provide a method of isolating their role. Contrary to that expectation, little evidence for hysteresis was obtained, for the outcomes of the ascending and descending series were highly similar. The failure to find such effects is puzzling, in view of the schedule-reversal effects noted by Charman and Davison. Presumably this discrepancy is due to the present ascending and descending series's involving more gradual changes that the common practice of complete reversals in schedules between successive conditions (e.g., in White et al., 1985). Whatever the cause of the discrepancy, it is clear that simple carry-over effects from the preceding condition are not an adequate explanation.

Table 5

Values of the Parameters from the Best Fits of Equation 1 to the Last Series of Conditions prior to the Change in Component

Duration, the Series with the 2-min Components, and the Final Series after the Return to the 10-sec Components

\begin{tabular}{ccccc}
\hline Subject & & $10 \mathrm{sec}$ & $2 \mathrm{~min}$ & $\begin{array}{c}10-\mathrm{sec} \\
\text { Return }\end{array}$ \\
\hline 81 & $a$ & .66 & .53 & .58 \\
& $b$ & .98 & .84 & .90 \\
83 & $a$ & .65 & .33 & .68 \\
& $b$ & .78 & 1.0 & .68 \\
84 & $a$ & .49 & .19 & .46 \\
& $b$ & 1.25 & 1.17 & 1.30 \\
85 & $a$ & .45 & .35 & .41 \\
& $b$ & .70 & .71 & .76 \\
\hline
\end{tabular}

Note-Only three conditions were used in each fit of the equation. Also note that Subjects 81 and 83 were trained on the low-value schedules and Subjects 84 and 85 were trained on the high-value schedules. 
Similar evidence pertaining to the role of hysteresis in undermatching effects in concurrent schedules has been provided by Davison and Hunter (1979).

Although Experiment 1 failed to demonstrate strong hysteresis effects, it did provide evidence that exposure to a long series of different schedule pairs attenuated sensitivity to the relative reinforcement rate. All subjects had lower sensitivities to the relative reinforcement rate during the later series of conditions than they did in the first series, and the pooled results (Table 4) showed a monotonic decline across successive conditions. A similar finding based on less extensive data was reported by Williams, (1983b), and it is also similar to that reported by Todorov, Castro, Hanna, Bittencourt de Sa, and Barreto (1983), using concurrent schedules. The latter authors noted that the exponent from the generalized matching law decreased from 1.07 (median of 5 subjects) for the first five presented conditions to .72 for Conditions 5-9. Why sensitivity to relative reinforcement rate is reduced by continued training is unclear, especially since successive conditions involve training to the same stability criterion. The finding is important, however, because it suggests that the notion of "steady-state" behavior, which is the foundation of much quantitative analysis of free-operant behavior, may be simplistic. It also may explain the disparity between previous results, since Charman and Davison (1982), who reported some of the lowest sensitivities to relative reinforcement rate with short-component multiple schedules, used the same subjects for a long series of conditions prior to beginning training with the shortest component durations. The effects of prior training also suggest the need to control carefully for the order of presentation when different component durations are being compared, which generally has not been done in previous studies.

The issue of major interest addressed by the present data is the effect of component duration on the sensitivity to the relative reinforcement rate, as assessed by the generalized matching law. Experiment 1 demonstrated that interactions with 10 -sec components were greater than those with 2-min components (see Table 5), an effect that resembles those reported in several previous studies on how component duration affects the absolute size of contrast effects (e.g., McSweeney, 1982). Thus, the observation by Charman and Davison (1982) that such effects occur only when the relative rate of reinforcement is held constant is not generally true.

The studies of Charman and Davison (1982) and White et al. (1985) did not result in an effect of component duration. The present study did. An important feature of the present data is that the absolute size of the exponent of the generalized matching law for the short-component schedules used here was substantially higher than previous estimates, especially when only those from the first series of exposures are considered. When consideration is limited to those conditions, the median value of the exponent was .74, in contrast to the median values of .3-.5 reported previously. Values in the latter range could sim- ply reflect the effects of reinforcement rate on response rate within a component, without any influence of the reinforcement rate in the alternative component (cf. Catania \& Reynolds, 1968). Such a possibility would suggest that the latter studies did not actually obtain contrast effects; consequently, there is no reason to expect that the degree of schedule "interaction," as assessed by Equation 1, should vary with component duration. The studies of Charman and Davison (1982) and White et al. (1985) do not allow an assessment of this issue, because they present no conditions in which the schedule of a given component was held constant while the schedule of the alternative component was varied between different values.

\section{EXPERIMENT 2}

The results of Experiment 1 show that the generalized matching law provides a sensitive method for capturing parametric variations in a multiple schedule. Indeed, one of the most impressive results of Experiment 1 was the accuracy of Equation 1: over $95 \%$ of the variance was accounted for in most cases, which suggests that the generalized matching law provides an excellent descriptive framework.

The accuracy of the fits of Equation 1 to the results with short-component schedules is somewhat surprising, when one considers that changes in component duration appear to be a composite of two separate effects, acting in opposite directions. Given a two-component schedule in which the components are correlated with different reinforcement rates, shorter durations of the higher-valued component (while the duration of the alternative component is constant) produce higher response rates in the highervalued component (Ettinger \& Staddon, 1982; Hinson et al., 1978). But when the duration of the higher-valued component remains constant while that of the lower-valued component (e.g., EXT) is varied, the highest response rate in the higher-valued component occurs when the alternative component is longer (Hinson \& Staddon, 1981; Schwartz, 1978; Wilton \& Clements, 1971). Since both of these variations occur simultaneously in the usual procedure, in which the duration of both components is varied together, their opposition should reduce any effect of component duration that is then evident.

If the usual effects of component duration are indeed composed of two different effects, this raises the issue of the relation between the two effects and whether or not they depend upon different processes. Previous research suggests that the two types of effects may have important functional differences. Although the effect of varying the duration of the alternative component on the response rate during a target component can be quite robust under some circumstances (Wilton \& Clements, 1971), in other studies it has disappeared with continued training (Hinson \& Staddon, 1981) or has failed to occur reliably (Ettinger \& Staddon, 1982). Variation of the duration of the target component itself, in comparison, has been shown to be robust across all phases of training (e.g., 
by Hinson et al., 1978). To the extent that these effects do indeed differ as a function of the degree of prior training, or other related variables, then component duration effects when the different effects are combined (as when the durations of all components are held equal) may be highly variable because of unequal contributions from the two effects at different points in training. Such variability might then be an important determinant of the conflict between previous studies (e.g., Charman \& Davison, 1982, vs. Shimp \& Wheatley, 1971).

In the present study, the question of whether the different types of component-duration effects are related to the distinction between different types of contrast was investigated. In previous work (Williams, 1979, 1981; Williams \& Wixted, 1986), it has been argued that contrast effects occurring in some target components have at least two separate constituents: contrast due to variation in the schedule in the component preceding the target (often labeled "local contrast"), and contrast due to variation in the schedule in the component following the target. The latter effect has generally been larger and been shown to increase over training, while the former effect has been highly variable across subjects and generally decreases with continued training. A differential relation of these two different effects to component duration is suggested by the results in Williams (1979). Williams found that component duration did not affect the role of the preceding schedule (because no consistent effect occurred with any component duration that was studied), but that the effects of the following schedule were increased by shorter components.

The present study contained a three-component (ABC) multiple schedule, in which the reinforcement rates in the first (A) and third (C) components were held constant at identical values while the reinforcement rate was varied in the middle (B) component. Previous investigations (Williams, 1981; Williams \& Wixted, 1986) have demonstrated that contrast effects are generally larger during Component $\mathrm{A}$, which precedes the locus of variation, than during Component $\mathrm{C}$, which follows it. At issue is how these differences are related to component duration. In one series of conditions, the duration of Component $B$ was held constant while the durations of $A$ and $C$ (which were always equal) were varied. In a second series, the duration of Components $A$ and $C$ was held constant while that of Component B was varied. In the final series, all three components were equal in duration and were varied together. The hypothesis was that response rate during Component $\mathrm{C}$ would be most affected by variation of Component $\mathrm{B}$, while response rate during Component $\mathrm{A}$ would be affected primarily by variation in itself.

\section{Method}

Subjects. Four White Carneaux pigeons were maintained at $80 \%$ of their free-feeding body weights by means of additional feeding after the end of the experimental sessions. All had served as subjects in an undergraduate laboratory, in which they had learned to keypeck under a variety of different simple schedules.
Apparatus. A standard one-key pigeon operant-conditioning chamber was constructed from a plastic picnic chest. The bird's chamber was approximately $30.5 \mathrm{~cm}$ in all dimensions. On the front panel were mounted a single pigeon key, $1.7 \mathrm{~cm}$ in diameter, which required a minimum force of $0.10 \mathrm{~N}$ for operation. The response key was located at the center of the panel, directly above a $5 \times$ $5 \mathrm{~cm}$ window through which the birds were fed when the food hopper was activated and illuminated with white light. Stimuli were projected onto the rear of the response key by a standard IEE 28-V in-line 12-stimulus projector. The stimuli used were a thin red $45^{\circ}$ line projected on a dark background, a thin white horizontal line also projected on a dark background, and a diffuse blue-green color. A shielded white houselight, illuminated at all times during experimental sessions, was located on the rear wall of the chamber directly opposite the intelligence panel.

Procedure. Because all subjects had prior experience with keypecking, they were begun immediately on the recycling threecomponent multiple schedule used throughout training. The stimuli for Components A, B, and C were initially the red oblique line, the green diffuse color, and the horizontal white line, respectively. During the first two sessions, the schedule for all three components was VI-30 sec, and the duration of each component was $1 \mathrm{~min}$. After these pretraining sessions, all subjects were begun immediately on the series of conditions shown in Table 6. Throughout this training, the schedules during Components $A$ and $C$ were always VI $2 \mathrm{~min}$. The schedule for Component B was either VI $30 \mathrm{sec}$ or VI $6 \mathrm{~min}$. The VI 30-sec and VI 2-min schedules were composed of 18 intervals from the distribution of Fleshler and Hoffman (1962), while the VI 6-min schedule was composed of 10 intervals. Separate schedules were used in each component, so that reinforcers that were arranged but not collected before a component terminated were held until the next presentation of that component.

Table 6

Order of Conditions in Experiment 2 and the Number of Sessions of Their Presentation

\begin{tabular}{|c|c|c|c|c|}
\hline Condition & Sessions & $\begin{array}{l}\text { Comp. B } \\
\text { Schedule }\end{array}$ & $\begin{array}{c}\text { Duration of } \\
\text { Comp. } A \text { and } C\end{array}$ & $\begin{array}{l}\text { Comp. B } \\
\text { Duration }\end{array}$ \\
\hline 1 & 33 & VI $30 \mathrm{sec}$ & 60 & 60 \\
\hline 2 & 33 & VI $30 \mathrm{sec}$ & 180 & 60 \\
\hline 3 & 30 & VI $6 \mathrm{~min}$ & 180 & 60 \\
\hline 4 & 30 & VI $6 \mathrm{~min}$ & 60 & 60 \\
\hline 5 & 30 & VI $6 \mathrm{~min}$ & 20 & 60 \\
\hline 6 & 26 & VI $30 \mathrm{sec}$ & 20 & 60 \\
\hline 7 & 34 & VI $30 \mathrm{sec}$ & 180 & 60 \\
\hline 8 & 25 & VI $6 \mathrm{~min}$ & 180 & 60 \\
\hline 9 & 25 & VI $6 \mathrm{~min}$ & 60 & 60 \\
\hline 10 & 39 & VI $6 \mathrm{~min}$ & 60 & 20 \\
\hline 11 & 22 & VI $30 \mathrm{sec}$ & 60 & 20 \\
\hline 12 & 27 & VI $30 \mathrm{sec}$ & 60 & 180 \\
\hline 13 & 25 & VI $6 \mathrm{~min}$ & 60 & 180 \\
\hline 14 & 20 & VI $6 \mathrm{~min}$ & 60 & 60 \\
\hline 15 & 24 & VI $30 \mathrm{sec}$ & 60 & 60 \\
\hline 16 & 21 & VI $30 \mathrm{sec}$ & 20 & 20 \\
\hline 17 & 21 & VI $6 \mathrm{~min}$ & 20 & 20 \\
\hline 18 & 21 & V1 $6 \mathrm{~min}$ & 180 & 180 \\
\hline 19 & 22 & VI $30 \mathrm{sec}$ & 180 & 180 \\
\hline 20 & 21 & VI $30 \mathrm{sec}$ & 60 & 60 \\
\hline 21 & 20 & VI $6 \mathrm{~min}$ & 60 & 60 \\
\hline 22 & 22 & VI $30 \mathrm{sec}$ & 60 & 60 \\
\hline 23 & 20 & VI $30 \mathrm{sec}$ & 60 & 180 \\
\hline 24 & 21 & VI $6 \mathrm{~min}$ & 60 & 180 \\
\hline 25 & 21 & VI 6 min & 20 & 20 \\
\hline 26 & 28 & VI $30 \mathrm{sec}$ & 20 & 20 \\
\hline
\end{tabular}

Note-Conditions 20-22 differed from all of the others in that the stimuli correlated with Components $A$ and $C$ were reversed. All durations are in seconds. 
As shown in Table 6, three types of variation of component duration were investigated. In the first series (Conditions 1-9), the duration of Component $B$ was held constant at $60 \mathrm{sec}$, while the durations of Components $A$ and $C$, which were always equal, were varied among $20 \mathrm{sec}, 60 \mathrm{sec}$, and $180 \mathrm{sec}$. For each of these durations, the schedule during Component $B$ was changed between VI $30 \mathrm{sec}$ and VI $6 \mathrm{~min}$. During the second series (Conditions 10-I5), the durations of Components $A$ and $C$ were held constant at $60 \mathrm{sec}$, and that of Component $B$ was varied among $20 \mathrm{sec}, 60 \mathrm{sec}$, and $180 \mathrm{sec}$. Once again the schedule during Component $B$ was varied between VI $30 \mathrm{sec}$ and VI 6 min for each component-duration value. During the third series (Conditions 14-19), all three components were varied simultaneously, again among 20,60 , and $180 \mathrm{sec}$. And once again the schedule during Component $B$ was varied between VI $30 \mathrm{sec}$ and VI $6 \mathrm{~min}$ for each component-duration value.

Conditions 20-22 were studied to evaluate the effects of the assignment of stimuli to components. For all other conditions, the stimulus assignment was always that the oblique red line occurred in Component A while the white horizontal line occurred in Component $C$. For the control series, these assignments were reversed. For these conditions, the duration of all three components was always $60 \mathrm{sec}$; the schedule for Component B was first VI $30 \mathrm{sec}$, then VI $6 \mathrm{~min}$, and again VI $30 \mathrm{sec}$. The final set of conditions (23-26) comprised replications of earlier conditions, to check for any systematic changes over the course of training.

Training during individual sessions continued for approximately $55 \mathrm{~min}$. Training within a condition continued until all subjects had attained a stability criterion. This was determined by calculating the relative response rates with respect to Components $A$ and $C$ in running blocks of three sessions. When these relative rates differed by no more than .05 , and when they showed no monotonically increasing or decreasing trends, the behavior was considered stable. All the subjects had to reach this criterion before they were moved together to the next condition. The actual number of sessions presented is shown in Table 6.

\section{Results}

The data to be presented were taken from the last nine sessions of each condition, after all the subjects had reached the stability criterion. Because there were multiple replications of some but not all conditions, the response rates from all presentations of a given condition were averaged together for the purposes of graphical presentation.

The top panel of Figure 3 shows the results of the third series, in which the duration of all three components was equal and varied together. Only the results from Components $\mathrm{A}$ and $\mathrm{C}$ are presented. The measure presented in Figure 3 is the size of the contrast effect in the two components, in percentage terms, when the reinforcement schedule during Component B was varied between VI $30 \mathrm{sec}$ and VI $6 \mathrm{~min}$. This measure was calculated through subtraction of the response rates during Components A and $\mathrm{C}$, respectively, when the schedule in Component $\mathrm{B}$ was VI $30 \mathrm{sec}$, from the corresponding rates in the two components when the schedule in Component B was VI $6 \mathrm{~min}$; the difference was then divided by the response rates when the schedule in Component B was VI 30.

Two major effects are evident in Figure 3. The size of the contrast effect was generally larger during Component $A$ than during Component $C$, and the size of the contrast effect decreased as component duration increased. These effects were tested for statistical reliability, using
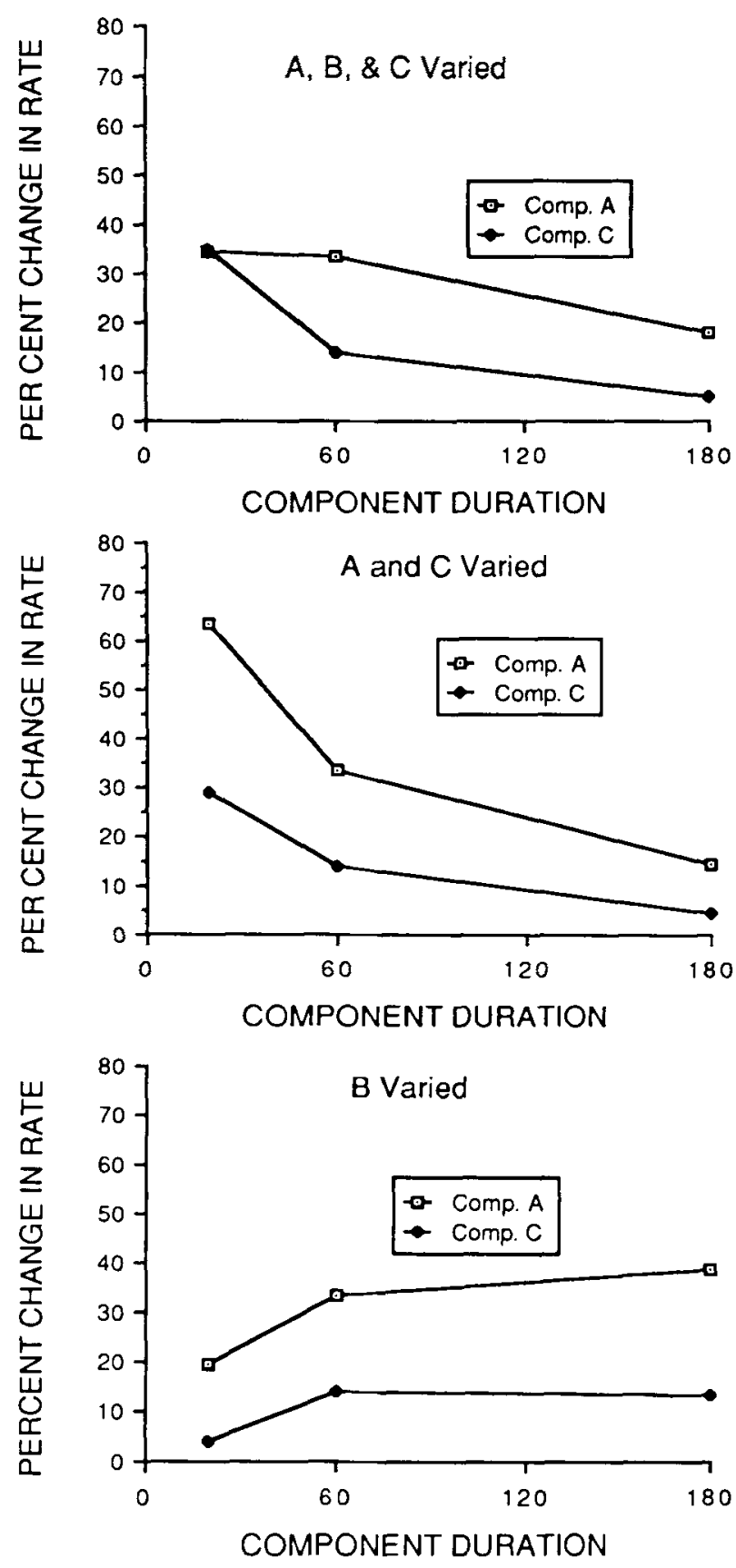

Figure 3. Contrast effects in Components $\mathrm{A}$ and $\mathrm{C}$ for the different types of variation in component duration. The designation at the top of each panel shows which components were varied in duration.

a two-way ANOVA (component $\times$ duration). The effect of the component location was significant $[F(1,3)=$ $17.86, p<.05$ ]; also significant was the effect of component duration $[F(2,6)=12.03, p<.01]$. But the interaction between the two variables was not signifiant $(F=1.46)$. An analysis of simple effects showed that the effects of component duration were significant for both Component $\mathrm{A}$ and Component $\mathrm{C}(p \mathrm{~s}<.05)$. 
The middle panel of Figure 3 shows the results from the series in which the duration of Component $B$ was held constant at $60 \mathrm{sec}$ and the durations of Components $A$ and $C$ were varied together. Contrast was again larger during Component $\mathrm{A}$, and for both components the size of the contrast effect decreased with longer components. The effect of component duration was consistent for all subjects for Component A, but more variable for Component $\mathrm{C}$, since 2 subjects failed to show any effect for that component. The results were again tested for statistical reliability with a two-way ANOVA (component $\times$ duration). The effect of Component $A$ as opposed to Component $C$ was again significant $[F(1,3)=22.99, p<.05]$, as was the effect of duration $[F(2,6)=7.85, p<.05]$. The interaction term was not significant $(F=1.45)$. Analysis of simple effects showed that the effect of component duration was significant for Component $\mathrm{A}$ ( $F=$ 14.45), but not for Component $C(F=1.42)$.

The bottom portion of Figure 3 shows the results when the durations of Components $A$ and $C$ were held constant at $60 \mathrm{sec}$ while the duration of Component $B$ was varied. In terms of the average results shown, the contrast effects increased in both target components with longer components. However, these results hide a large amount of variability among subjects, since only 2 subjects showed the pattern that was seen, while 1 subject showed no effect, and a 4th subject showed an effect in the opposite direction. For all subjects, however, the response rate in Component $\mathrm{A}$ was higher than in Component $\mathrm{C}$. The results were again analyzed statistically with a two-way ANOVA. The comparison of Components $\mathrm{A}$ and $\mathrm{C}$ was again significant $[F(1,3)=12.11, p<.05]$, but the effect of component duration $(F<1)$ and of the interaction $(F<1)$ were not significant.

All of the results heretofore described came from conditions in which the same stimulus assignments were maintained across conditions. Because the degree of contrast may vary with the degree of stimulus similarity (Williams, 1988), it is possible that the differences in the degree of contrast between Components $\mathrm{A}$ and $\mathrm{C}$ could have been due to the stimulus variables rather than their locations relative to Component $\mathbf{B}$. To control for this possibility, three conditions (20-22) were studied, in which the stimulus assignments for Components $\mathrm{A}$ and $\mathrm{C}$ were reversed. During the first and third of these conditions, the schedule during Component B was VI $30 \mathrm{sec}$, whereas during the second it was VI $6 \mathrm{~min}$. For all three of these conditions, component duration in all components was held constant at $60 \mathrm{sec}$. This allowed an assessment of the degree of contrast with this new stimulus assignment, which could then be compared to the corresponding contrast values seen with the opposite assignment. Figure 4 shows the results of this analysis.

In general, the effects of stimulus assignment were negligible. The mean size of the contrast effect during Component $\mathrm{C}$ was increased $2 \%-3 \%$, and that during Component $\mathrm{A}$ was decreased by a similar amount; but these effects were not consistent across subjects. A two-

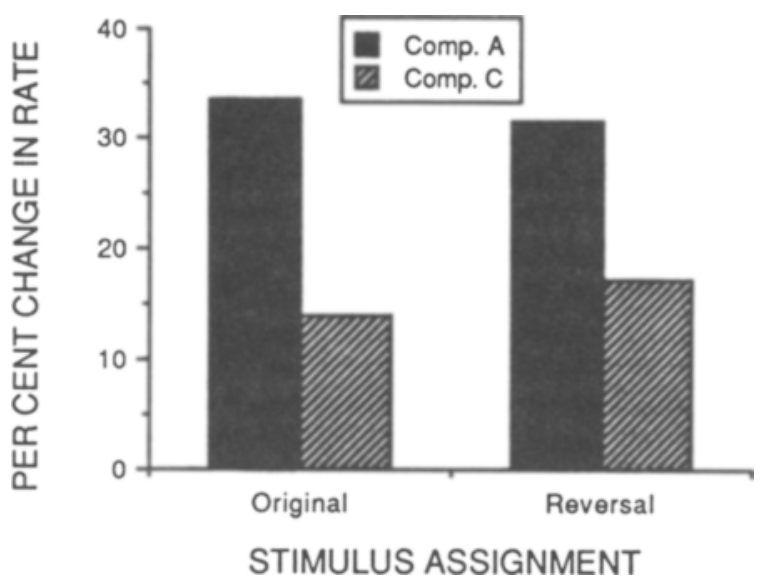

Figure 4. Contrast effects in Components $A$ and $C$ for the conditions in which all components were $60 \mathrm{sec}$ in duration. The different pairs of bars correspond to reversed stimulus assignments for the two components.

way ANOVA (component $\times$ stimulus assignment) revealed that the effect of component location continued to be significant $[F(1,3)=24.96, p<.05]$, whereas neither the effect of stimulus $(F<1)$ nor the interaction $(F<1)$ was significant. Thus, the pattern of results seen in Figure 3 appears to be independent of the stimuli assigned to Component $\mathrm{A}$ as opposed to Component $\mathrm{C}$.

\section{Discussion}

The present results replicated the previous finding that contrast is larger with shorter component durations when the components of the schedule are equal in length (e.g., in McSweeney, 1982), as well as the finding that contrast is greater with shorter target components when the duration of the target component is varied while holding constant the duration of the alternative component(s) (e.g., see Hinson et al., 1978). The results further replicated the previous finding that contrast is greater in a target component followed by the locus of reinforcement variation than in a target component preceded by the point of variation (cf. Williams \& Wixted, 1986).

The present results are also similar to those other studies of the pattern of variability. With respect to contrast in a target component preceded by the point of reinforcement variation (in this case, Component $\mathrm{C}$ ), previous work with three-component schedules has shown that only 1 of 4 subjects showed any substantial contrast effect in that location regardless of component duration (Williams, 1981; Williams \& Wixted, 1986). Similarly, when a fourcomponent schedule was studied such that two target components were preceded by extinction as opposed to a higher-valued VI, (Williams, 1979, Experiment 1), a higher rate following extinction occurred for only 1 of 4 subjects, and this effect was not affected by component duration (over a range of 15 to $180 \mathrm{sec}$ ). In contrast, Wilton and Clements (1971) reported a robust effect of the duration of a preceding $S-$, although it should be noted that they presented their subjects with a single cycle per 
session, with $\mathbf{S}$ - durations substantially longer than those investigated here. Finally, Hinson and Staddon (1981) reported an effect of the duration of the preceding component early in training, but this effect disappeared with continued training. Why such effects occur in some situations and not in others remains uncertain.

The fact that the duration of the preceding component produced weaker and less consistent effects than did the duration of the target component itself should not be surprising, because otherwise there would be no rationale for why contrast should vary with component duration when the duration of all components was equal. Given that contrast is strongest with short durations of the target component and with long durations of the alternative component, these effects should be in opposition when the durations of both components are equal. The fact that contrast is nevertheless larger with shorter components even with equal durations implies that the effect of the duration of the target component must be larger. Given that there appear to be some circumstances, albeit not well understood, when the duration of the alternative component does play an important role (see, e.g., Wilton \& Clements, 1971), the further implication is that failures to find any effect of component duration, when the durations of all components are varied together, may not be due to the insensitivity to the component duration per se, but rather to the averaging of the two types of effects.

Evidence for the opposing natures of the two types of effects can be seen by comparing the relative sizes of contrast effects for the different types of component-duration variations. If the effects with equal-duration components (Figure 1, top panel) were a combination of the effects when the target and alternative components were varied separately (middle and bottom panels, respectively), then those with equal durations should be reduced relative to those when the durations of only the target components were varied. Thus, the contrast effects seen in the middle panel should be larger than those seen in the top panel. This was true for Component $\mathrm{A}$, but behavior during Component $C$ is less clear, primarily because of the variability across subjects in the degree of contrast during Component $\mathrm{C}$, which is noted in the results.

Whatever the causes of the variability seen in this and in previous studies, the present results make clear that the usual method of studying component duration, with all components equal in length, is deceptively simple. The present findings suggest that at least two different effects are conflated with such a procedure, and that an adequate analysis requires that they be analyzed separately. The fact that the generalized matching law describes the results accurately (e.g., see Experiment 1) despite this combination of separate effects suggests that mere descriptive power does not justify any conclusions about underlying processes.

A final issue addressed by the present study is the relation between the different types of component-duration effects and the contrast effects occurring in the preceding as opposed to the following target components. The results provide no evidence of any interaction between the type of component duration and the effects of the preceding as opposed to the following schedules, because in all cases the patterns of change were similar for Components $A$ and $C$, the only difference being that the effects in Component A were larger. The implication of this similarity for interpreting the relation beween the two types of effects is uncertain, since other studies have suggested that they depend upon different mechanisms. Similarity in results as a function of component-duration variation does not exclude that possibility, for there is no reason in principle that different mechanisms should not be affected similarly by that variable.

In summary, the schedule interactions in multiple schedules are a systematic function of the duration of the schedule components, when assessed both with the sensitivity exponent of the generalized matching law and according to changes in response rate (contrast effects). However, the usual procedure used to study such effects, when all components are of equal duration and are varied together, is misleading, because it confounds two opposing effects, which differ in both magnitude and variability across subjects. Thus, a complete analysis of multiple-schedule interactions must depend upon understanding the separate processes underlying these different effects.

\section{REFERENCES}

BAUM, W. M. (1974). Chained concurrent schedules: Reinforcement as situation transition. Journal of the Experimental Analysis of Behavior, 22, 91-101.

Catania, A. C., \& Reynolds, G. S. (1968). A quantitative analysis of responding maintained by interval schedules of reinforcement. Journal of the Experimental Analysis of Behavior, 11, 327-383.

Charman, L., \& Davison, M. (1982). On the effects of component durations and component reinforcement rates in multiple schedules. Journal of the Experimental Analysis of Behavior, 37, 417-439.

Davison, M. C., \& Hunter, I. W. (1979). Concurrent schedules: Undermatching and control by previous experimental conditions. Journal of the Experimental Analysis of Behavior, 32, 233-244.

EtTinger, R. H., \&TAddon, J. E. R. (1982). Behavioral competition, component duration and multiple-schedule contrast. Behaviour Analysis Letters, 2, 31-38.

Fleshler, M., \& HoffMAN, H. S. (1962). A progression for generating variable-interval schedules. Journal of the Experimental Analysis of Behavior, 5, 529-530.

Hinson, J. M., Malone, J. C., JR., McNally, K., \& Rowe, D. (1978). Effects of component length and of the transitions among components in multiple schedules. Journal of the Experimental Analysis of Behavior, 29, 3-16.

Hinson, J. M., \& STAddon, J. E. R. (1981). Some properties of local contrast. Behaviour Analysis Letters, 1, 275-281.

McLean, A. P., \& WHITE, K. G. (1983). Temporal constraint on choice: Sensitivity and bias in multiple schedules. Journal of the Experimental Analysis of Behavior, 39, 405-426.

MCSweEney, F. K. (1982). Positive and negative contrast as a function of component duration for key pecking and treadle pressing. Journal of the Experimental Analysis of Behavior, 37, 281-293.

MCSweeney, F. K., Farmer, V. A., Dougan, J. D., \& Whipple, J. E. (1986). The generalized matching law as a description of multipleschedule responding. Journal of the Experimental Analysis of Behavior, 45, 83-101.

SchWARTz, B. (1978). Stimulus-reinforcer contingencies and local behavioral contrast. Joumal of the Experimental Analysis of Behavior, 29, 297-308. 
Shimp, C. P., Wheatley, K. L. (1971). Matching to relative reinforcement frequency in multiple schedules with a short component duration. Joumal of the Experimental Analysis of Behavior, 15, 205-210.

Spealman, R. D. (1976). Interactions in multiple schedules: The role of the stimulus-reinforcer contingency. Journal of the Experimental Analysis of Behavior, 26, 79-93.

Stevens, S. S. (1957). On the psychophysical law. Psychological Review, 64, 153-181.

Todorov, J. C. (1972). Component duration and relative response rates in multiple schedules. Journal of the Experimental Analysis of Behavior, 17, 45-49.

Todorov, J. C., Castro, J., hanna, E., Bittencourt de Sa, M., \& Barreto, M. (1983). Choice, experience, and the generalized matching law. Journal of the Experimental Analysis of Behavior, 40, 99-111.

White, K. G., Pipe, M.-E., Mclean, A. P., \& Redman, S. (1985). Temporal proximity and reinforcement sensitivity in multiple schedules. Journal of the Experimental Analysis of Behavior, 44, 207-215.

Williams, B. A. (1979). Contrast, component duration, and the following schedule of reinforcement. Journal of Experimental Psychology: Animal Behavior Processes, 5, 379-396.

Williams, B. A. (1980). Contrast, signaled reinforcement, and the relative law of effect. American Journal of Psychology, 93, 617-629.
WiLliams, B. A. (1981). The following schedule of reinforcement as a fundamental determinant of steady state contrast in multiple schedules. Joumal of the Experimental Analysis of Behavior, 35, 293-310.

WILLIAMS, B. A. (1983a). Another look at contrast in multiple schedules. Joumal of the Experimental Analysis of Behavior, 39, 345-384.

WiLliams, B. A. (1983b). Overmatching in multiple schedules. Behaviour Analysis Letters, 3, 171-182.

WiLliams, B. A. (1988). The effects of stimulus similarity on different types of behavioral contrast. Animal Learning \& Behavior, 16, 206-216.

Williams, B. A., \& Fantino, E. (1978). Effects on choice of reinforcement delay and conditioned reinforcement. Journal of the Experimental Analysis of Behavior, 29, 77-86.

Williams, B. A., \& WixTed, J. T. (1986). An equation for behavioral contrast. Joumal of the Experimental Analysis of Behavior, 45, 47-62.

Wilton, R. N., \& Clements, R. O. (1971). Behavioral contrast as a function of the duration of an immediately preceding period of extinction. Journal of the Experimental Analysis of Behavior, 16, 425-428.

(Manuscript received June 28, 1988 ; revision accepted for publication October 18,1988 .) 